\title{
Gruppenpraxen sind ein Megatrend - ist die Therapiefreiheit gesichert?
}

Heinrich Zürcher

Leiter Kommunikation Argomed Ärzte AG
Als Hausärzteorganisation stellen wir fest: Gruppenpraxen sind ein Megatrend. Überall schiessen sie aus dem Boden. Die Vorteile sind evident: geringere Investitionen, bessere Ausnützung der Einrichtungen, Delegation der Administration, Möglichkeit zur Teilzeitarbeit, kollegialer Austausch, keine zu langfristige Bindung. Aber wie steht es mit der Therapiefreiheit? Aus medizinischen und ethischen Gründen sind Ärzte und Patienten auf eine möglichst grosse Entscheidungsfreiheit angewiesen. Mit Einschränkungen aus wirtschaftlichen Gründen haben wir uns mehr oder weniger abgefunden. Müssen Ärzte in einer Gruppenpraxis auch zusätzlich in Kauf nehmen, dass deren Eigentümer auf die Behandlung Einfluss nehmen? Wer mich bezahlt, dessen Interessen muss ich vertreten?

\section{Verschiedene Investoren - spezifische Interessen}

Investoren sind oft Krankenversicherer, kommerzielle Investoren, Ärzte und Ärztegruppen, zunehmend auch Spitäler. Neben einem gewissen Gewinn, der legitim ist, streben sie weitere Ziele an: Krankenversicherer betreiben HMOs mit dem Ziel möglichst geringer Behandlungskosten. Kommerzielle Investoren suchen möglichst hohen Gewinn. Spitäler brauchen Zulieferer für ihre ambulanten und stationären Angebote. Ärzte sind in diesen Praxen angestellt und müssen sich an Weisungen ihrer Arbeitgeber halten.

\section{Ärzte als Eigentümer einer Gruppenpraxis}

Solche Abhängigkeiten lassen sich vermeiden, wenn die Ärzte selbst ihre Gruppenpraxis besitzen. So können sie die Ziele ihres Unternehmens selbst bestimmen. Unerwünschte Einflussnahmen externer Geldgeber lassen sich vermeiden. Die Finanzierung solcher Praxen kann zwar schwierig sein, ist aber lösbar, wenn sich etablierte Hausärzte mit Neueinsteigern zusammenschliessen. Dabei können sich Jungärzte zuerst anstellen lassen oder sich mit geringen Beiträgen einkaufen, um ihre Beteiligung später aufzustocken. Solche Praxen sind von Anfang an gut ausgelastet und wirtschaftlich erfolgreich. Argomed empfiehlt deswegen allen Interessenten, sich einer ärzteeigenen Gruppenpraxis anzuschliessen. Die geringere Fluktuation der Ärzte in solchen Praxen beweist, dass die
Arbeitszufriedenheit höher ist als in Praxen mit einem Anstellungsverhältnis.

Argomed und andere ärzteeigene Organisationen erhalten laufend Anfragen um Unterstützung bei der Gründung von Gruppenpraxen. Gefragt sind Beratung und Begleitung von Hausärzten bei der Firmengründung, oft beim Erstellen von Business- und Projektplänen sowie deren Umsetzung als Projektleiter. In einzelnen Fällen hat Argomed Mandate für die Unterstützung in betriebswirtschaftlichen und organisatorischen Fragen nach der Betriebsaufnahme erhalten, weil Ärzte sich auf die medizinische Tätigkeit konzentrieren wollen. An den Gruppenpraxen «Doktor Zentrum» hat sich Argomed finanziell beteiligt.

\section{Ohne gute Software geht es nicht}

Für den Erfolg einer Gruppenpraxis ist die richtige Software unabdingbar. Sie vereinfacht und beschleunigt die Praxisprozesse und ist Voraussetzung für die interne Koordination und Kommunikation. Die elektronische Krankengeschichte (elKG) mit ihrer Leserlichkeit und dauernden Verfügbarkeit hat schlagende Vorteile gegenüber der papierbasierten KG. Die Software dient aber nicht nur der Dokumentation, sondern ist Praxisinformationssystem mit vielen weiteren Funktionen: Erstellen von Rezepten und Zeugnissen, Integration von Labordaten und Befunden, integriertem Textsystem, Administration mit Verrechnung und Rechnungswesen, Agenda, Dokumentenverwaltung, Datenaustausch mit externen Institutionen, zuverlässige Archivierung.

\section{Sicherheit und Zuverlässigkeit}

Gerade im Gesundheitswesen muss die Praxisinformatik äusserst sicher und immer verfügbar sein. Das bedingt kurze Interventionszeiten bei Störungen. Datenverlust und Datendiebstahl müssen in jedem Fall ausgeschlossen werden. Die Auswahl des IT-Partners mit Erfahrung und Referenzen bedarf besonderer Beachtung. Bisher stehen Lösungen mit lokalem Server und Praxis-Netzwerk im Vordergrund. Das bewährt sich, bedeutet aber einen gewissen Aufwand für Wartung, Updates und Störungsbehebung. Der Trend geht in Richtung Web-Lösungen. Dabei werden Software und Daten bei einem spezialisierten Anbieter gespeichert. Der Zugriff geschieht mit einem gewöhnlichen Computer über den Internet-Browser. Dies ermöglicht auch den einfachen Zugriff von zu Hause oder auf Hausbesuch. Die Anwender müssen sich nicht mehr um Updates und die Datensicherheit kümmern. Die externen Kosten sind bei diesen Lösungen allerdings höher. 